\title{
Atividade física, condições de saúde e classificação econômica das mulheres atendidas por treinamento personalizado
}

A cada dia a sociedade busca uma qualidade de vida, em especial as mulheres que pensando em obter um futuro saudável e ter uma velhice mais adequada, tendo em vista que mesmo com a globalização e a tecnologia cada vez mais avançada que acaba levando a comodidade, a praticidade e o tempo ficam mais corridos com: as atividades diárias, trabalho, estudos, família, ou seja, as mesmas vivem buscando se sentir bem e estar bem. O objetivo do presente estudo é identificar a classe econômica, o nível de atividade física e condições de saúde de mulheres atendidas por treinamento personalizado. A população do presente estudo trata de mulheres praticantes de musculação de uma academia personalizada na cidade de Juazeiro do Norte/CE. A amostra foi composta por 33 mulheres, com faixa etária entre 18 a 60 anos. Para a coleta dos dados foi utilizado o Questionário Internacional de Atividade Física (IPAO), o Pentáculo do Bem-Estar (PBE). Para definir a posição financeira na sociedade, foi usado o critério classificação econômica. Os dados foram analisados por meio de estatística descritiva através do software Bem-Estar (PBE). Para definir a posição financeira na sociedade, foi usado o critério classificação econômica. Os dados foram analisados por meio de estatística descritiva através do software
JASP. Como resultados podemos destacar que as mulheres de classe social mais alta tendem a ter índices mais elevados de qualidade de vida geral em relação as classes mais baixas, as

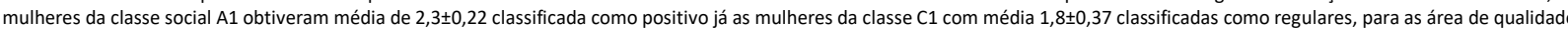
de vida analisadas separadas, destaca-se os resultados para o controle de estresse que não houve associação entre as classes, porém, encontra-se com índice regulares. Na relação entre classe social e nível de atividade física as mulheres das classes mais baixas são fisicamente mais ativas que as mulheres de classe mais alta, em C1 temos 3 mulheres (75\%) classificadas como muito ativa e na classe A1 encontramos apenas 1 mulher $(16,7 \%)$ na mesma classificação. Tendo como considerações que a relação entre a classe social pode interferir para escolha e pratica de atividade física, porém, pode-se também fazer com que tenha um declínio da mesma por conta das atividades de status social, afetando ainda na área de controle de estresse como foi apresentado nos dados dessa pesquisa.

Palavras-chave: Atividade física; Mulheres; Classe Econômica.

\section{Physical activity, health conditions and economic classification of women attended by personalized training}

\begin{abstract}
Every day, society seeks a quality of life, especially women who are thinking about achieving a healthy future and have a more adequate old age, considering that even with globalization and the and are doing well. The objective of the present study is to identify the economic class, the level of physical activity and health conditions of women attended by personalized training. The population of the present study are women who practice weight training at a personalized gym in the city of Juazeiro do Norte/CE. The sample consisted of 33 women, aged between 18 and population of the present study are women who practice weight training at a personalized gym in the city of Juazeiro do Norte/CE. The sample consisted of 33 women, aged between 18 and
60 years. For data collection, the International Physical Activity Questionnaire (IPAQ), the Pentacle of Well-Being (PBE) was used. And to define the financial position in society, the economic classification criterion was used. The data were analyzed using descriptive statistics using the JASP software. As a result we can highlight that women of higher social class tend to have highe levels of general quality of life in relation to lower classes, women of social class A1 had an average of $2.3 \pm 0.22$ classified as positive already class C1 women with an average of $1.8 \pm 0.37$ classified as regular, for the areas of quality of life analyzed separately, the results for stress control stand out, as there was no association between the classes, however it is found with regular indexes. In the relationship between social class and level of physical activity, women from the lower classes are physically more active than women from the upper class, in C1 we have 3 women (75\%) classified as very active and in class $A 1$ we find only 1 woman (16.7\%) in the same classification. Considering that the relationship between the social class can interfere with the choice and practice of physical activity, however, of this research.
\end{abstract}

Keywords: Physical activity; Women; Economic class.

Topic: Educação em Saúde

Reviewed anonymously in the process of blind peer.
Received: $\mathbf{1 3 / 0 3 / 2 0 2 0}$

Approved: 14/05/2020
José de Caldas Simões Neto (iD)

Centro Universitário Dr. Leão Sampaio, Brasil http://lattes.cnpq.br/0470733825644726

http://orcid.org/0000-0003-1036-2315

josedecaldas@leaosampaio.edu.br

Graciane Gomes da Silva (iD)

Centro Universitário Dr. Leão Sampaio, Brasil http://lattes.cnpq.br/0099806223014695

http://orcid.org/0000-0003-3827-2362

graciane00@gmail.com

Marcos Antonio Araujo Bezerra (D)

Centro Universitário Dr. Leão Sampaio, Brasil

http://lattes.cnpq.br/4643352879633283

http://orcid.org/0000-0002-3385-4024

marcosantonio@leaosampaio.edu.br
Lara Belmudes Bottcher (iD)

Centro Universitário Dr. Leão Sampaio, Brasil

http://lattes.cnpq.br/4112151773664370

http://orcid.org/0000-0002-5504-4102

larabottcher@leaosampaio.edu.br
Referencing this:

SIMÕES NETO, J. C.; SILVA, G. G.; BEZERRA, M. A. A.; BOTTCHER, L. B. Atividade física, condições de saúde e classificação econômica das mulheres atendidas por treinamento personalizado. Educationis, v.8, n.2, p.41-48, 2020. DOI: http://doi.org/10.6008/CBPC23183047.2020.002.0006 


\section{INTRODUÇÃO}

A cada dia a sociedade busca uma qualidade de vida, em especial as mulheres que pensando em obter um futuro saudável e ter uma velhice mais adequada, tendo em vista que mesmo com a globalização e a tecnologia cada vez mais avançada que acaba levando a comodidade, a praticidade e o tempo ficam mais corridos com: as atividades diárias, trabalho, estudos, família, ou seja, as mesmas vivem buscando se sentir bem e está bem. Conforme o tempo passa o corpo sofre bastante transformação.

Os maus hábitos e dizer q não tem tempo leva ao sedentarismo que é um grande precursor de risco, podendo levar a morte, pode-se definir sedentário a falta de atividade física, ou seja, pessoas que em sua atividade diária gastam poucas calorias e que alimenta os comportamentos inadequados para uma vida saudável, podendo destacar: passar a maior parte do tempo sentado, assistir televisão, usar aparelho celular, transporte que facilita para se deslocar mais rápido de um lugar para o outro e acabam ocupando o seu tempo livre que poderia ser destinados a bons hábitos saudáveis.

A qualidade de vida enquanto uma abordagem quantitativa se refere a recursos materiais, mas é bastante ampla, pois a mesma aborda desde as necessidades básicas de cada indivíduo, o seu sentimento de bem-estar até a sua capacidade de desenvolver seu potencial dentro da sociedade em que a mesma está inserida (HORTA et al., 2013).

De acordo com Russo (2005), a procura de um padrão de beleza corporal, vem sendo como uma meta a ser conquistada, principalmente nessas grandes cidades. Hoje em dia a mídia é o maior mercado de como ficar bonita, ela impõe um modelo a ser seguido como, por exemplo, perna grande, cintura fina e abdômen trincado, como se o corpo fosse uma massa de modelar que fica do jeito que a pessoa quer, custem o que custar. Com isso a procura de tratamentos estéticos, cirurgias, produtos de beleza, academias e de opções que levem ao corpo ideal é crescente.

Dessa forma surge a necessidade de não somente ter um corpo belo, mais ter uma qualidade de vida, a grande procura nesse caso é a atividade física, que vai proporcionar vários benefícios tais como: melhora do stress, trabalha a socialização, diminui o percentual de gordura, aumenta a massa muscular, melhora do sistema cardiorrespiratório, obtém qualidade no sono, promove o bem-estar, aumenta a autoestima e previne várias doenças, dentre outros benefícios.

A partir daí surge, na educação física, o treinamento personalizado, que tem como principal objetivo desenvolver os trabalhos de acordo com as individualidades de cada sujeito/cliente, para assim atingir os seus objetivos com mais rapidez sem fugir da qualidade do treino e sempre levando em consideração a necessidade de cada cliente. Dando uma maior assistência, segurança e melhores resultados. A proposta é atrair cada vez mais as pessoas para a prática de exercícios físicos (CASTRO, 2009).

Os clientes que procuram um atendimento personalizado, ainda é pouco, por conta da questão financeira, pois as academias convencionais o custo é bem abaixo do valor cobrado pelo personal training, a grande massa que busca esse atendimento é de classe média. Que tem recurso financeiro para desfrutar desse trabalho. Para melhores resultados à procura de outros profissionais da saúde, nutricionista, 
fisioterapeutas e os médicos. A atividade física pode ser ponderada como a melhor forma de se obter a saúde pública com baixo custo, em promover benefícios, que a mesma traz o combate ao sedentarismo. Pois além de prevenir doenças, ela as trata da mesma possibilitando viver melhor (PARDINI et al., 2001).

Com a sociedade contemporânea, utiliza-se cada vez menos das capacidades corporais, a inatividade física é fator determinante para uma vida construída de maus hábitos, com isso promover mudanças no seu que diz respeito a estilo de vida é a base para uma nova expectativa saudável. Estudo baseado nessa temática vem adquirindo grande relevância, para colaborar e agregar valores a sociedade de que exercitar gera melhorias em todos os aspectos sociais e econômicos (ASSUMPÇÃO et al., 2002). A importância maior dessa pesquisa é relacionar a classe econômica, o nível de atividade física e condições de saúde de mulheres atendidas por treinamento personalizado, procurando identificar qual a classe de mulheres procura esse atendimento.

Sabe-se dos inúmeros benefícios da prática de atividade física relacionada a saúde, ao bem-estar e a qualidade de vida, assim como o surgimento de doenças relacionadas ao sedentarismo. Assim, o presente estudo, torna-se de fundamental importância para contribuir na melhoria da promoção de saúde e também para quebrar as barreiras para que todas as classes possam ter acesso a um treinamento personalizado. Dessa forma, as clientes interessadas por um atendimento personalizado são em grande parte de classe média, com o objetivo cuidar do corpo, a fins estéticos e buscam a qualidade de vida, promoção de saúde e melhoria do condicionamento físico e também por conta da acessibilidade de horário que é marcado para cada duas alunas uma profissional de educação física.

Assim, veio o interesse de se investigar se classe baixa da população tem acesso ao treinamento personalizado. Nesse contexto, o presente estudo teve como principal objetivo identificar a classe econômica, o nível de atividade física e condições de saúde de mulheres atendidas por treinamento personalizado.

\section{MATERIAIS E MÉTODOS}

O estudo proposto, refere-se a uma pesquisa de campo, do tipo exploratório com caráter descritivo em uma abordagem predominantemente quantitativa. A população do presente estudo foi composta por mulheres praticantes de musculação de uma academia personalizada na cidade de Juazeiro do Norte/CE e sua amostra composta por 33 mulheres, com faixa etária entre 18 a 60 anos. Foi utilizado como critérios de inclusão da pesquisa: a) ser mulher; b) está matriculado com atendimento personalizado; c) ser maior de 18 anos. Como critério de exclusão: a) está com a matrícula cancelada e b) ser maior de 60 anos.

Foi iniciado o estudo através da solicitação de autorização junto a proprietária da academia, através de um ofício elaborado pelo professor orientador do trabalho, para realização da pesquisa, com as mulheres praticantes da musculação por treinamento personalizado. Na qual foi informada dos objetivos e metodologia do referido estudo.

As clientes que aceitarem participar do estudo assinaram o Termo de Consentimento Livre e Esclarecido (TCLE), contendo todas as informações da acerca da pesquisa, conforme prevê a Resolução no 
466/12 do Concelho Nacional de Saúde. A referida resolução assegura todos os princípios éticos estabelecidos para a realização de pesquisa envolvendo humanos, submetido e aprovado no comité de ética do Centro Universitário Dr. Leão Sampaio com número de parecer 2.449.178.

Para a obtenção dos níveis de atividade foi utilizado o Questionário Internacional de Atividade Física (IPAQ) que classifica os sujeitos em muito ativo, ativo, irregularmente ativo $A$ e $B$, sedentário, para o estilo de vida individual o Pentáculo do Bem-Estar (PBE), que inclui as particularidades da nutrição, controle do stress, atividade física diária, relacionamento social e comportamentos preventivos, sendo demonstrada em figura no formato do Pentáculo, podendo ser classificadas com índice Negativo menor de 1; índice Regular entre 1 e 1,99 e Positivo entre 2 e 3 (NAHAS, 2001). Para definir a posição financeira na sociedade, foi usado o critério classificação econômica, o Critério de Classificação Econômica Brasil, que enfatiza sua função de estimar o poder de compra das pessoas e famílias urbanas, abandonando a pretensão de classificar a população em termos de 'classes sociais' em A1, A2, B1, B2 C1, C2 D e E, sendo A1 classe mais alta e E mais baixa. Os dados foram tabulados no programa Excel 2013 e posteriormente analisados de forma descritiva no programa JASP 8.4.0 por média, desvio padrão e por distribuição de frequência relacionando a classe econômica, com o nível de atividade física e a qualidade de vida.

\section{RESULTADOS E DISCUSSÃO}

De acordo com os dados obtidos, mediante a aplicação dos questionários do pentáculo do bem estar, do IPAQ e o critério classificação econômica, apresentados os resultados a seguir com as associações entre a classe social e qualidade de vida na tabela 1 , podemos destacar que as mulheres de classe social mais alta tendem a ter índices mais elevados de qualidade de vida geral em relação as classes mais baixas, as mulheres

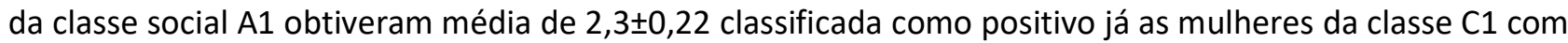

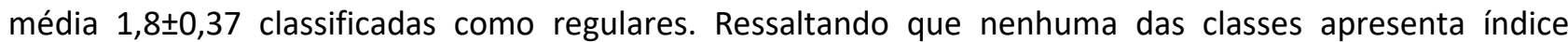
negativos de qualidade de vida geral.

Analisando as cinco áreas do pentáculo do bem estar essa relação entre classe social e qualidade de vida se mantem no campo da nutrição com $A 1$ com resultados melhores e positivos de $2,7 \pm 0,56$ em relação as classe mais baixas como $\mathrm{C} 1$ com índices positivos de 2,2 $\pm 0,33$; para o campo da atividade física $\mathrm{A} 1$ obteve

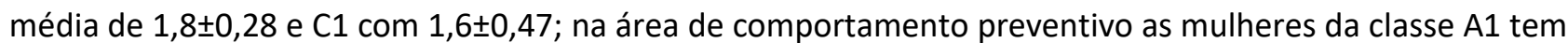

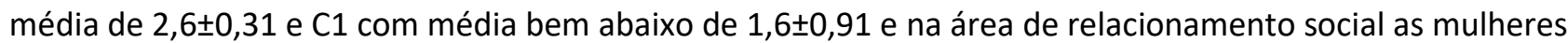

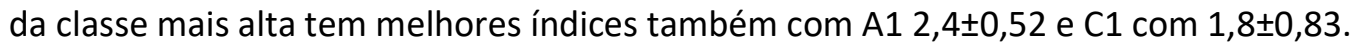

Para a área relacionada ao controle de estresse, não encontramos uma relação maior para as

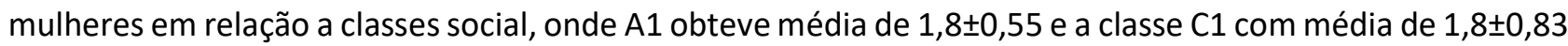
apresentando maior desvio padrão, e ambas classificadas como regulares de acordo com o pentáculo do bem-estar. Dressler et al. (1998) utilizaram o conceito de "estilo de vida" de modo diferenciado, como componentes para diferenças sócio-econômicas-culturais, em que um "modelo padrão" do estilo de vida foi determinado pela tendência central da amostra para cada grupo estudado, na cidade de Ribeirão Preto no estado de São Paulo. Os grupos sociais foram denominados de Favela a classe social mais baixa, Conjunto 
Habitacional a classe baixa, Tradicional para a classe média baixa e Alta para a classe média.

O resultado do estudo presente que estes 'modelos padrões' se apresentaram inversamente associados à sintomas depressivos e percepção global de estresse e quando confrontados com os indicadores categoria ocupacional, educação e salário, verificou-se que a educação $(p<0,05)$ e com o salário $(p<0,01)$, e os sintomas depressivos e a percepção de estresse associasse inversamente, muito significativamente com sintomas depressivos $(\beta=-236, p<0,01)$ e estresse globalmente percebido $(\beta=-358, p<0,01)$ sendo uma possível relação entre o controle de estresse encontrado em nosso estudo em relação as classes sociais mais elevadas na pesquisa, já que a amostra é de maioria de classe média e alta.

Tabela 1: Descrição dos dados referente a qualidade de vida das mulheres atendidas por treinamento individualizado pelas áreas classificadas pelo pentáculo do bem-estar e classe social econômica.

\begin{tabular}{|c|c|c|c|c|c|c|c|c|c|c|c|c|c|c|c|}
\hline & \multicolumn{5}{|c|}{ Nutrição } & \multicolumn{5}{|c|}{ Atividade Física } & \multicolumn{5}{|c|}{ Comp. Preventivo } \\
\hline & A1 & A2 & B1 & B2 & C1 & A1 & A2 & B1 & B2 & C1 & A1 & A2 & B1 & B2 & C1 \\
\hline $\mathbf{N}$ & 6 & 8 & 8 & 7 & 4 & 6 & 8 & 8 & 7 & 4 & 6 & 8 & 8 & 7 & 4 \\
\hline$M$ & 2.7 & 2.1 & 1.9 & 1.9 & 2.2 & 1.8 & 2.0 & 2.0 & 1.9 & 1.6 & 2.6 & 2.5 & 2.3 & 2.1 & 1.6 \\
\hline DP & 0.56 & 0.39 & 0.43 & 0.46 & 0.33 & 0.28 & 0.48 & 0.57 & 0.48 & 0.47 & 0.31 & 0.60 & 0.44 & 0.64 & 0.91 \\
\hline \multicolumn{6}{|c|}{ Relacionamento Social } & \multicolumn{5}{|c|}{ Controle de Estresse } & \multicolumn{5}{|c|}{ Qualidade de Vida Geral } \\
\hline $\mathbf{N}$ & A1 & A2 & B1 & B2 & C1 & A1 & A2 & B1 & B2 & C1 & A1 & A2 & B1 & B2 & C1 \\
\hline$M$ & 6 & 8 & 8 & 7 & 4 & 6 & 8 & 8 & 7 & 4 & 6 & 8 & 8 & 7 & 4 \\
\hline DP & 2.4 & 2.6 & 2.5 & 2.1 & 1.8 & 1.8 & 2.0 & 2.2 & 1.9 & 1.8 & 2.3 & 2.2 & 2.2 & 1.9 & 1.8 \\
\hline $\mathbf{N}$ & 0.52 & 0.48 & 0.40 & 0.59 & 0.83 & 0.55 & 0.75 & 0.86 & 0.87 & 0.83 & 0.22 & 0.23 & 0.19 & 0.32 & 0.37 \\
\hline
\end{tabular}

Legenda: $\mathrm{N}=$ número de participantes; $\mathrm{M}=$ Média; $\mathrm{DP}=$ Desvio Padrão.

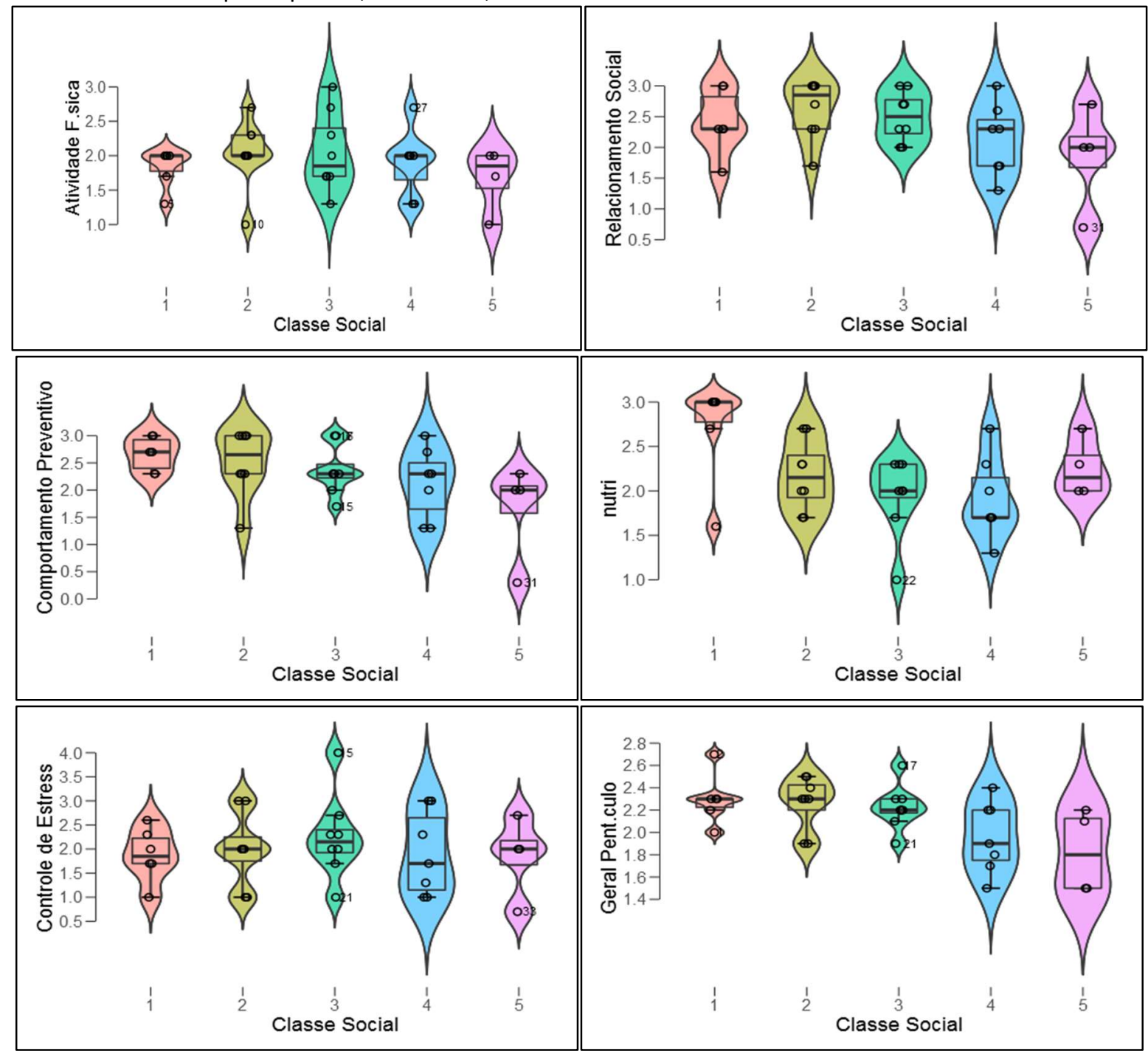


Tabela 2: Níveis de atividade física das mulheres atendidas por treinamento individualizado relacionados com a classe social econômica

\begin{tabular}{|c|c|c|c|}
\hline Classe Social & Nível de Atividade Física & $(\mathrm{N})$ & $(\%)$ \\
\hline \multirow[t]{4}{*}{ A1 } & Muito Ativo & 1 & 16.7 \\
\hline & Ativo & 4 & 66.6 \\
\hline & Irregularmente Ativo B & 1 & 16.7 \\
\hline & Total & 6 & 100.0 \\
\hline \multirow[t]{4}{*}{$A 2$} & Muito Ativo & 4 & 50.0 \\
\hline & Ativo & 4 & 50.0 \\
\hline & Irregularmente Ativo B & 0 & 0.0 \\
\hline & Total & 8 & 100.0 \\
\hline \multirow[t]{4}{*}{ B1 } & Muito Ativo & 3 & 37.5 \\
\hline & Ativo & 5 & 62.5 \\
\hline & Irregularmente Ativo B & 0 & 0.0 \\
\hline & Total & 8 & 100.0 \\
\hline \multirow[t]{4}{*}{ B2 } & Muito Ativo & 3 & 42.9 \\
\hline & Ativo & 4 & 57.1 \\
\hline & Irregularmente Ativo B & 0 & 0.0 \\
\hline & Total & 7 & 100.0 \\
\hline \multirow[t]{4}{*}{ C1 } & Muito Ativo & 3 & 75.0 \\
\hline & Ativo & 1 & 25.0 \\
\hline & Irregularmente Ativo B & 0 & 0.0 \\
\hline & Total & 4 & 100.0 \\
\hline
\end{tabular}

Na tabela 2 descreve os resultados da relação entre as classes social e o nível de atividade física em relação ao IPAQ, os dados foram analisados frente as cinco classes da amostra encontradas nessa pesquisa, podemos destacar que as mulheres das classes mais baixas são fisicamente mais ativas que as mulheres de classe mais alta, em C1 temos 3 mulheres (75\%) classificadas como muito ativa e na classe A1 encontramos apenas 1 mulher (16,7\%) na mesma classificação.

No estudo de Matsudo et al. (2008) que avaliou o nível de atividade física na população do estado de São Paulo, com entrevistados 2001 indivíduos de 14 a 77 anos de idade, sendo 953 do sexo masculino e 1048 do sexo feminino, destaca a classificação do nível de atividade física segundo o nível socioeconômico, evidenciou que os Grupos A (mais ricos) e E (mais pobres) apresentaram maior prevalência de indivíduos que não alcançaram a recomendação, sendo o fato mais evidente no Grupo $\mathrm{E}$.

Já no estudo de Mazo et al. (2005) que verificou o nível de atividade física e a sua relação com as características sócio demográficas e as condições de saúde de 1.980 mulheres idosas com 65 anos ou mais de idade, na cidade de Florianópolis, não encontrou uma diferença estatisticamente significativa entre o nível de atividade física e classe econômica com ( $p=0.057)$. Em um estudo sobre a atividade física em 64.101 mulheres americanas de Adams-Campbell et al. (2000) observaram que a atividade física vigorosa aumenta em relação ao a nível educacional, mas a não pratica de atividade física modera e ou caminhada, os autores ainda destacam para o reflexo dos hábitos de pratica de exercícios física serem reflexos dos conhecimentos a partir de aulas de Educação Física em ambientes educacionais.

Partindo ainda para as discussões referente a pratica de atividade física e níveis educacionais, DiezRoux et al. (1999) em sua pesquisa associou a prevalência de fatores de risco cardiovasculares e variáveis sócio demográficas em 695 indivíduos na comunidade de Harlen, na cidade de Nova lorque - EUA, e observaram que os rendimentos e o nível educacional associam-se inversamente à inatividade física nas mulheres, com significância estatística de $\mathrm{p}<0,05$ e $\mathrm{P}<0,001$ respectivamente. Essa relação se dá pelas 
mulheres desse grupo terem nível superior completos, assim podendo ter um reflexo para a pratica de atividade física.

Já Luc Boltanki (1989; citado por PALMA, 2017) em uma análise realizada na França sobre a relação das classes sociais e o corpo, destaca que a prática de atividade física declina com decresce a classe social, onde foi classificado por porcentagem as categorias profissionais em relação a não realização de práticas de esportes destacando para as categorias de Agricultores com 70\%, Operários 53\%, comerciantes $44 \%$, Assalariados 42\% dirigentes de Nível Média 42\% e Difringentes Assalariados com 32\%.

\section{CONCLUSÕES}

O presente estudo foi desenvolvido com uma população específica de mulheres em um studio de musculação exclusivo para esse o atendimento na cidade de Juazeiro do Norte, no qual observou a classe econômica, qualidade de vida e nível de atividade física. A classe que predominou nessa pesquisa, com os resultados mais satisfatório foi a classe $A 1$, em que as mulheres que possuem recursos financeiro maior pera classificação geral da qualidade de vida, sendo que quanto menor a classe social tende a diminuir os índices de qualidade de vida. Vale ressaltar que os índices apresentados mesmo diminuindo entre as classes sociais são resultados regulares e positivos.

A partir essa análise das condições de saúde das mulheres com a classe social se manteve o mesmo padrão em quatro área avaliadas pelo pentáculo, Nutrição, Atividade Física, Comportamento Preventivo e Relacionamento Social. No Controle de Estresse não encontramos associação referente a classe social, onde as classes aqui estudantes obtiveram resultados semelhantes. Verificamos também que o nível de atividade física teve relação pequena relação inversa ao resultado anterior quanto a classe social, mostrando que as mulheres quanto mais bem estabelecida financeiramente são menos ativas em relação as mulheres de menos classe social.

Em relação as condições de saúde as mulheres dessa pesquisa estão com uma qualidade de vida com índices positivos, podendo a classe econômica interferi positivamente nesse ponto, já que elas buscam e tem com uma maior facilidade atendimento de profissionais especializados para realizar seu acompanhamento, físico, nutricional e psicológico. Podemos assim avaliar que a classe social pode interferir na qualidade de vida das mulheres. Considerando as análises aqui exposta, ao finalizar o estudo entendemos que essa temática deve ser mais aprofundada afim de melhorar buscar novas análises e relações, bem como realizar outras amostras para comparação de dados entre as populações.

\section{REFERÊNCIAS}

ADAMS-CAMPBELL, L.; ROSENBERG, L.; WASHBURN, R.; RAO, R.; KIM, K.; PALMER, J.. Descritive epidemiology of physical activity in African-American women. Preventive Medicine, v.30, p.43-50, 2000.

ASSUMPÇÃO, L. OT; MORAIS, P. P.; FONTOURA, H.. Relação entre atividade física, saúde e qualidade de vida. Notas Introdutórias. Revista Digital, v.8, n.52, p.1-3, 2002.
CASTRO, S. C.. O perfil do cliente do profissional de educação física que atua como personal trainer na região metropolitana de Belo Horizonte. Universidade Federal de Minas Gerais, 2009.

DIEZ-ROUX, A.; NORTHRIDGE, M.; MORABIA, A.; BASSETT, M; SHEA, S.. Prevalence and social correlates of cardiovascular disease risk factors in Harlen. American Journal of Public Health, v.89, n.3, p.302-307, 1999. 
DRESSLER, W. W.; BALIEIRO, M. C.; SANTOS, J. E.. Culture, socioeconomic status, and physical and mental health in Brazil. Medical Anthropology Quarterly, v.12, n.4, p.424446, 1998.

HORTA, P. M.. Qualidade de vida entre mulheres com excesso de peso e doenças crônicas não transmissíveis. Rev. Gaúcha Enferm., v.34, n.4, p.121-129, 2013.

NAHAS, M. V.. Atividade física, saúde e qualidade de atividade física, saúde e qualidade de vida: conceito vida: conceitos e sugestões para um estilo de vida e sugestões para um estilo de vida ativo. 2 ed. Londrina, 2001.

MATSUDO, S. M.; MATSUDO, V. R.; ARAÚJO, T.; ANDRADE, D.; ANDRADE, E.; OLIVEIRA, L.; BRAGGION, G.. Nível de atividade física da população do Estado de São Paulo: análise de acordo com o gênero, idade, nível socioeconômico, distribuição geográfica e de conhecimento. Revista Brasileira de Ciência e Movimento, v.10, n.4, p.41-50, 2008.
MAZO, G. Z.; MOTA, J.; GONÇALVES, L. H. T.; MATOS, M. G.. Nível de atividade física, condições de saúde e características sociodemográficas de mulheres idosas brasileiras. Revista Portuguesa de Ciências do Desporto, v.5, n.2, p.202-212, 2005.

PALMA, A.. Atividade física, processo saúde-doença e condições socioeconômicas: uma revisão da literatura. Revista Paulista de Educação Física, v.14, n.1, p.97-106, 2017.

PARDINI, R.; MATSUDO, S.; ARAÚJO, T.; MATSUDO, V.; ANDRADE, E.; BRAGGION, G.; ANDRADE, D.; OLIVEIRA, L.; FIGUEIRA, A.; RASO, V.. Validação do questionário internacional de nível de atividade física (IPAQ - versão 6): estudo piloto em adultos jovens brasileiros, Rev. Bras. Ciên. e Mov. Brasília, v.9, n.3, p.45-51, 2001.

RUSSO, R.. Imagem corporal: construção através da cultura do belo. Revista Movimento \& Percepção, Espírito Santo de Pinhal, v.5, n.6, 2005.

A CBPC - Companhia Brasileira de Produção Científica (CNPJ: 11.221.422/0001-03) detém os direitos materiais desta publicação. Os direitos referem-se à publicação do trabalho em qualquer parte do mundo, incluindo os direitos às renovaç̃oses, expansões e disseminações da contribuição, bem como outros direitos subsidiários. Todos os trabalhos publicados eletronicamente poderão posteriormente ser publicados em coletâneas impressas sob coordenação da Sustenere Publishing, da Companhia Brasileira de Produção Científica e seus parceiros autorizados. Os (as) autores (as) preservam os direitos autorais, mas não têm permissão para a publicação da contribuição em outro meio, impresso ou digital, em português ou em tradução. 